\title{
From an electrophoretic mobility shift assay to isolated transcription factors: a fast genomic-proteomic approach
}

\author{
Astrid R Mach-Aigner ${ }^{1}$, Karin Grosstessner-Hain ${ }^{3}$, Marcio J Poças-Fonseca² ${ }^{2}$ Karl Mechtler ${ }^{3}$, Robert L Mach ${ }^{1 *}$
}

\begin{abstract}
Background: Hypocrea jecorina (anamorph Trichoderma reesei) is a filamentous ascomycete of industrial importance due to its hydrolases (e.g., xylanases and cellulases). The regulation of gene expression can influence the composition of the hydrolase cocktail, and thus, transcription factors are a major target of current research. Here, we design an approach for identifying a repressor of a xylanase-encoding gene.

Results: We used streptavidin affinity chromatography to isolate the Xylanase promoter-binding protein 1 (Xpp1). The optimal conditions and templates for the chromatography step were chosen according to the results of an electrophoretic mobility shift assay performed under repressing conditions, which yielded a DNA-protein complex specific to the AGAA-box (the previously identified, tetranucleotide cis-acting element). After isolating AGAA-box binding proteins, the eluted proteins were identified with Nano-HPLC/tandem MS-coupled detection. We compared the identified peptides to sequences in the H. jecorina genome and predicted in silico the function and DNA-binding ability of the identified proteins. With the results from these analyses, we eliminated all but three candidate proteins. We verified the transcription of these candidates and tested their ability to specifically bind the AGAA-box. In the end, only one candidate protein remained. We generated this protein with in vitro translation and used an EMSA to demonstrate the existence of an AGAA-box-specific protein-DNA complex. We found that the expression of this gene is elevated under repressing conditions relative to de-repressing or inducing conditions.

Conclusions: We identified a putative transcription factor that is potentially involved in repressing xylanase 2 expression. We also identified two additional potential regulatory proteins that bind to the xyn2 promoter. Thus, we succeeded in identifying novel, putative transcription factors for the regulation of xylanase expression in H. jecorina.
\end{abstract}

\section{Background}

Hypocrea jecorina (anamorph Trichoderma reesei, [1]) is an abundant filamentous ascomycete. $H$. jecorina breaks down polysaccharides with a variety of hydrolytic enzymes that act synergistically $[2,3]$. Due to the high secretory capacity of this fungus (up to $100 \mathrm{~g} / \mathrm{L},[4]$ ), $H$. jecorina has gained industrial importance and is employed both in the fermentative production of native extracellular enzymes and heterologous protein

\footnotetext{
* Correspondence: rmach@mail.zserv.tuwien.ac.at

'Gene Technology, Department of Gene Technology and Applied Biochemistry, Institute of Chemical Engineering, TU Wien, Getreidemarkt 9/166/5/2, A-1060 Wien, Austria

Full list of author information is available at the end of the article
}

production. The hydrolases that are secreted by this fungus are applicable to many industries, including textiles (e.g., [5]), food and feed, (e.g., [6-8]), paper (e.g., [9,10]) and, most recently, biofuel production [11-13].

In 2006, we reported the identification of the main activator of hydrolases in $H$. jecorina, Xyr1 (Xylanase regulator 1) [14]. In addition to Xyr1, two transcription factors, Ace1 (Activator of cellulases 1) and Ace2 (Activator of cellulases 2 ), are potentially involved in the regulation of hydrolases in $H$. jecorina $[15,16]$. These two additional narrow domain transcription factors seem to directly modulate the mode of action of the general regulator Xyr1 $[17,18]$. The Carbon catabolite repressor Cre1 [19] has also been described as a wide domain repressor of

\section{Ciomed Central}


particular hydrolase-encoding genes (e.g., [20,21]). Some years ago it was also postulated that another putative repressor protein exists that is specific for $x y n 2$ (xylanase 2) [22]. An in vivo genomic footprinting approach identified the AGAA-box within the $x y n 2$ promoter as a relevant cis-acting motif that is protected under glucose repressing conditions. Until recently, however, the corresponding trans-acting factor was not known.

Geyer and co-workers showed for the restriction endonuclease MboI that protein-DNA complexes could be identified with metal affinity chromatography, followed by mass spectrometry [23]. Later, affinity chromatography-SELEX (systematic evolution of ligands by exponential enrichment) was reported to be suitable for isolating transcription factors and modelling proteinDNA interactions [24,25]. In 2005, a streptavidin affinity assay was used to purify proteins of a known size that bind to the AP-1 (Activator protein-1) sequence (p47 and $\mathrm{p} 49$ ) in human cancer cells; these proteins were subsequently identified by sequencing the SDS-PAGE (sodium dodecylsulphate - polyacrylamide gel electrophoresis) protein bands [26].

Herein, we describe the isolation and identification of a transcription factor of unknown size with the combination of streptavidin affinity chromatography, a NanoHPLC (high performance liquid chromatography)-tandem MS (mass spectrometry)-coupled detection system, and a genome based allocation. Our method is a fast genomic-proteomic approach for the identification of new and completely unknown transcription factors starting from a protein-DNA complex identified with an electrophoretic mobility shift assay (EMSA).

\section{Results}

The AGAA-motif within the xyn2 promoter is bound under glucose-repressing conditions

In vivo genomic footprinting data indicate that the transcriptional regulation of $x y n 2$ expression involves the following cis-acting elements: a CCAAT-box, two Xyr1binding motifs (GGGTAA and GGCTGG) known to bind Xyr1 (Xylanase regulator 1, [14]), and an AGAA-box $[18,22]$. Figure 1 summarizes the architecture of the $x y n 2$ promoter. The AGAA-box, as well as the CCAAT-box and one Xyr1-binding motif, are located on the antisense strand. The AGAA tetranucleotide is fully protected under non-inducing conditions [22]. Cell-free extract protein from $H$. jecorina transferred to repressing (glucose) or inducing (xylan) conditions was subjected to an EMSA using radiolabeled probes. A protein-DNA complex was readily observed using cell-free extract obtained under repressing conditions and a short oligonucleotide containing the AGAA-box (Pxyn2a, see Table 1) (Figure 1). No protein-DNA interaction was detected when cell-free extracts from inducing conditions were used (Figure 1),

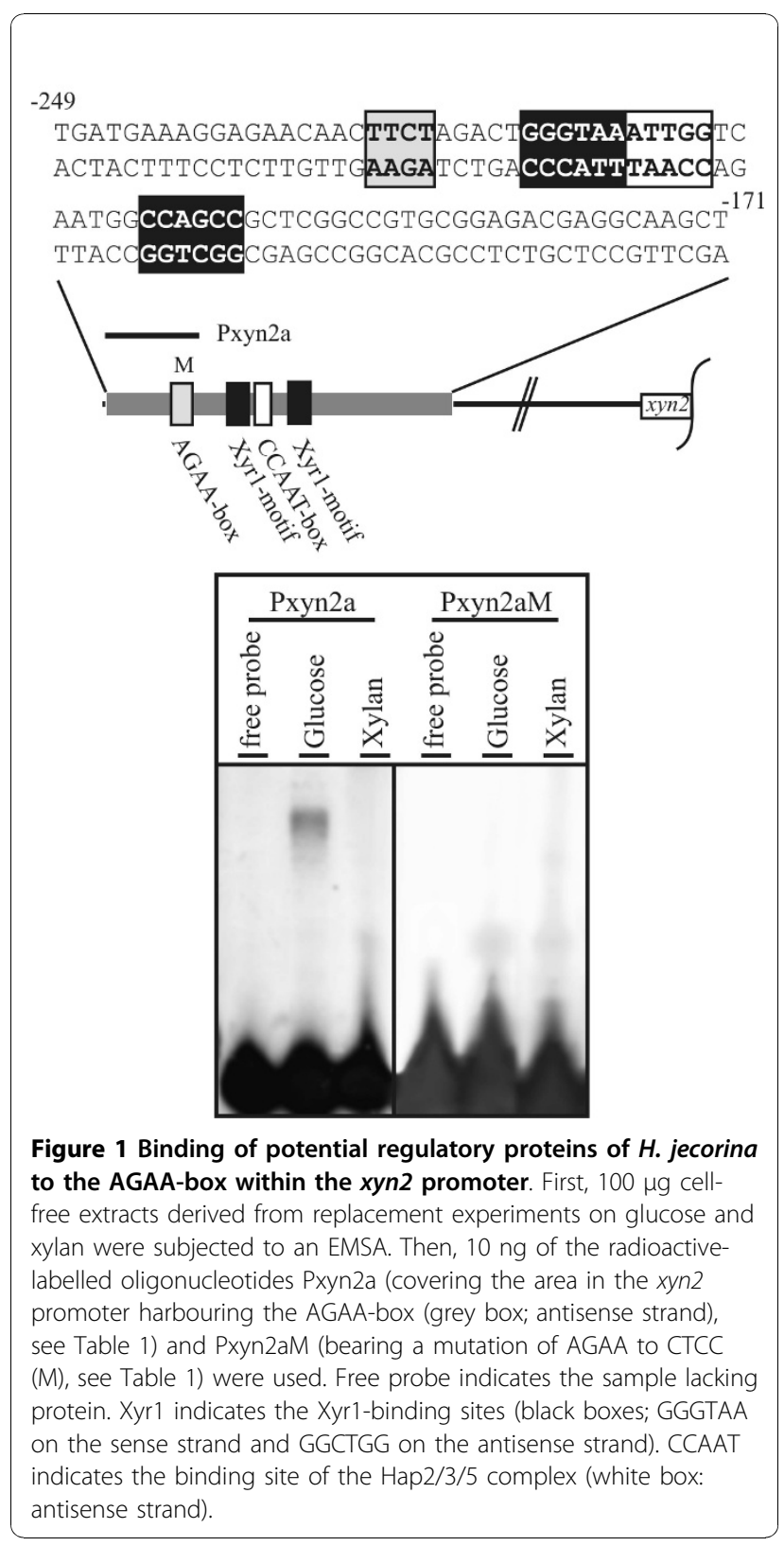

supporting the idea that this part of the promoter is involved in repression. If the AGAA sequence is mutated CTCC (Pxyn2aM, see Table 1), no DNA-protein complex is formed under either repressing or inducing conditions (Figure 1). This result indicates that this part of the $x y n 2$ promoter is essential for binding a transcription factor under repressing conditions.

\section{Streptavidin-based isolation and identification of AGAA- binding proteins}

The DNA-protein complex observed in the EMSA includes a putative repressor of $x y n 2$ transcription (termed “Xpp1", Xylanase promoter-binding protein 1). 
Table 1 Oligonucleotides used in this study

\begin{tabular}{|c|c|c|}
\hline Name & Sequence $\left(5^{\prime}-3^{\prime}\right)$ & Employment \\
\hline Bxyn2p250f & Biotin-TGATGAAAGGAGAACAACTTCTAGACTG & Affinity Chromatography \\
\hline Bxyn2p250r & CAGTCTAGAAGTTGTTCTCCTITCATCA & Affinity Chromatography \\
\hline CKT067 & CACTCCACATGTTAAAGGCGCATTCAACCAGCTTC & EMSA/Affinity Chromatography \\
\hline СКТ068 & GAAGCTGGTTGAATGCGCCTITAACATGTGGAGTG & EMSA/Affinity Chromatography \\
\hline Exp2488F & GGATCCGACCGCATGGCGCACAAC & Construction of p2488DBD \\
\hline Exp2488R & CTCGAGTCAACAGAATCCTCTCGGGTCG & Construction of p2488DBD \\
\hline Exp3151F & GGATCCGAAGAAACCGCCAAGGCGC & Construction of p3151DBD \\
\hline Exp3151R & CTCGAGAGATGTGTACGTCGGGTTTC & Construction of p3151DBD \\
\hline Exp7236F & GGATCCACACACGACCCCAACGCC & Construction of p7236DBD \\
\hline Exp7236R & CTCGAGCGCGAGGGGGTTTCCATTC & Construction of p7236DBD \\
\hline$f \mid \operatorname{tn} 2488 f$ & GGTACCATGGCACAAGCCCTCGACATTTCC & Construction of p2488 \\
\hline fltn2488r & GCGGCCGCTCAACAGAATCCTCTCGGGTCGAAG & Construction of p2488 \\
\hline LPxyn2f-FAM & FAM-TGATGAAAGGAGAACAACTTCTAGACTGGGTAAATTGGTCAATGGCCAGCCGCTC & FAM-labelled EMSA \\
\hline LPxyn2r & GAGCGGCTGGCCATTGACCAATTTACCCAGTCTAGAAGTTGTTCTCCTTTCATCA & FAM-labelled EMSA \\
\hline LPxyn2Mf-FAM & FAM-TGATGAAAGGAGAACAACGGAGAGACTGGGTAAATTGGTCAATGGCCAGCCGCTC & FAM-labelled EMSA \\
\hline LPxyn2Mr & GAGCGGCTGGCCATTGACCAATTTACCCAGTCTCTCCGTTGTTCTCCTTTCATCA & FAM-labelled EMSA \\
\hline Pxyn2af & TGATGAAAGGAGAACAACTTCTAGACTG & Radioactive EMSA \\
\hline Pxynzar & TGACCAGTCTAGAAGTTGTTCTCCTTTC & Radioactive EMSA \\
\hline Pxyn2aMf & $\overline{\text { TGATGAAAGGAGAACAACGGAGAGACTG }}$ & Radioactive EMSA \\
\hline Pxyn2aMr & TGACCAGTCTCTCCGTTGTTCTCCTTTC & Radioactive EMSA \\
\hline transkr2488f & $\overline{A G C T T C C A C A A A C A T G A C G C C G}$ & Transcript analysis \\
\hline transkr2488r & CATGGCGATTTCGAGCAGTCG & Transcript analysis \\
\hline transkr3500f & СTCTTCAGGTCCTTATGAAGGTCG & Transcript analysis \\
\hline transkr3500r & GAGTAGCTGTCCGATCCACG & Transcript analysis \\
\hline transkr3151f & GATGTCTGAGGAATCTTCAAGCGC & Transcript analysis \\
\hline transkr3151r & GGAGTCTTGCTTCGATTGCGG & Transcript analysis \\
\hline transkr7236f & GTGTACCTGGACCTTGCGC & Transcript analysis \\
\hline transkr7236r & CTGCTTCTCCTGGGGCG & Transcript analysis \\
\hline
\end{tabular}

The employment of the oligonucleotides used in this study is given. Underlined bases represent introduced restriction enzyme sites or bases added for labelling. Double underlined bases indicate introduced point mutations.

To isolate this complex, the same batch of cell-free extracts from $H$. jecorina transferred to glucose was subjected to affinity chromatography. A schematic of the procedure is shown in Figure 2. Biotinylated versions of the same oligonucleotides used for the EMSA (Bxyn2p250, see Table 1) were incubated on ice with streptavidin beads (Figure 2). Under the EMSA reaction conditions, this mixture was incubated with the cell-free extract, which should include Xpp1 (Figure 2). After magnetic separation, the whole eluate was subjected to Nano-HPLC separation. The eluted peptides were monitored on the HPLC at a wavelength of $214 \mathrm{~nm}$ and directly applied to the mass spectrometer.

The tandem mass spectra were analyzed in silico as described above. 81 eluted proteins (see additional file 1) were identified and analyzed further with FGENESH V1 and GENEWISE 1. Three proteins with DNA-binding domains were identified and represent potential transcription factors (Table 2). These proteins (scan number 2488 (protein ID 122879), 3151 (protein ID
21557), 7236 (protein ID 108909)) were investigated in more detail and are hereafter referred to as "2488prp" (prp, promotor-binding protein), "3151prp", and "7236prp". The mass spectra for these three selected candidate genes are available online at: http://cores. imba.oeaw.ac.at $/$ index.php?id=3731.

To verify that the genes encoding 2488prp, 3151prp, and 7236prp correspond to transcripts in H. jecorina, we performed RT-PCR using cDNAs obtained under inducing and non-inducing conditions. Using the primers listed in Table 1, we could clearly detect transcripts for the genes 2488prp, 3151prp, and 7236prp (Table 2).

\section{Cloning, expression, and binding of putative AGAA- binding transcription factors of $H$. jecorina}

Since transcripts for all three genes encoding the putative regulatory proteins were detected, the respective DNA-binding domains (DBD) were cloned and expressed as GST-fusion proteins using the pGEX-4T- 


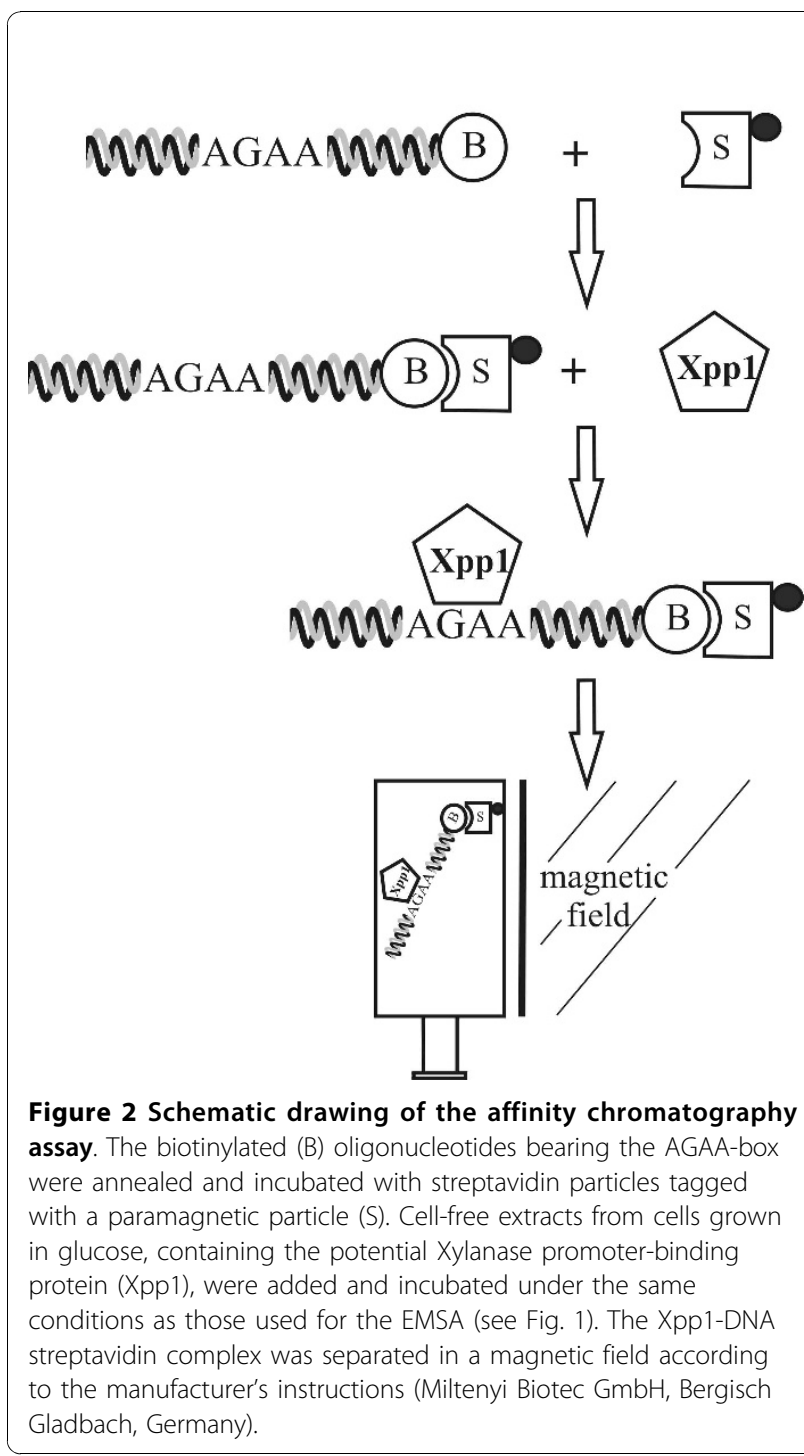

2 vector and E. coli BL21-Gold. Production of the recombinant proteins was verified by SDS-PAGE (Figure 3A). All clones, two for the expression of the 3151prp DBD, three for the expression of the 7236prp DBD, and two for the expression of the 2488prp DBD, produced proteins of expected sizes $(34 \mathrm{kD}$ for

Table 2 Isolated and identified $\boldsymbol{H}$. jecorina potential DNAbinding proteins of interest and verification of their transcript formation under repressing conditions in strain QM9414

\begin{tabular}{llc}
\hline $\begin{array}{l}\text { Protein } \\
\text { ID }\end{array}$ & Predictions according to FGENESH & Transcription \\
\hline 2488 & $\begin{array}{l}\text { Putative DNA-binding protein, helix-loop- } \\
\text { helix }\end{array}$ & + \\
3151 & $\begin{array}{l}\text { DNA-binding domain, Zn-finger; BRAHMA- } \\
\text { complex }\end{array}$ & + \\
7236 & DNA-binding "high mobility group" protein & + \\
\hline
\end{tabular}

A

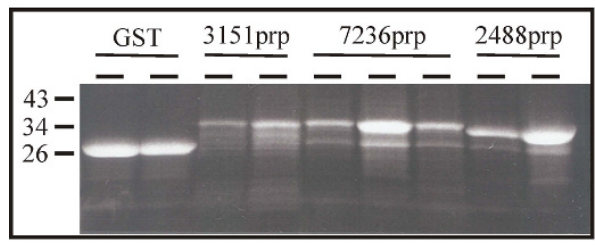

B

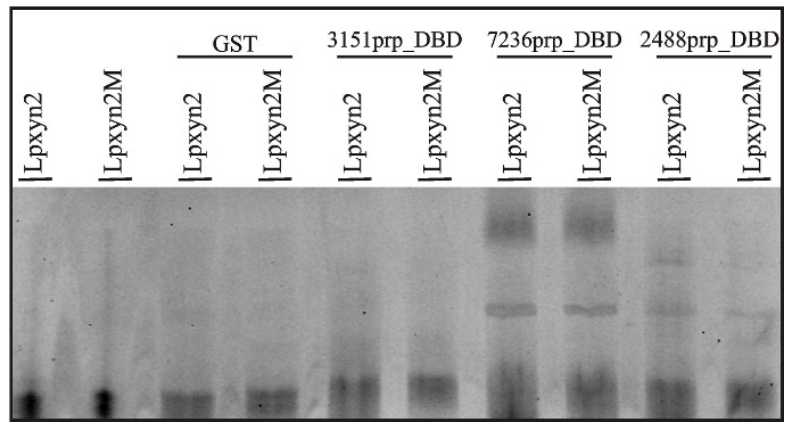

Figure 3 Expression and binding of DNA-binding domains (DBD) of putative repressor proteins to the AGAA-box within $\boldsymbol{x y n} 2$ promoter of $\boldsymbol{H}$. Jecorina. (A) SDS-PAGE of two clones of the DBD of 3151, three clones of the DBD of 7236, and two clones of the DBD of 2488. All clones heterologously expressed the GSTfusion proteins (3151 prp, 7236prp, 2488prp). GST, expression of the GST-protein alone, as a control. A prestained protein ladder (Fermentas) was used. (B) EMSA with $100 \mathrm{ng}$ of the DBDs expressed as GST-fusion proteins (see Fig. 3A). Finally, 15 ng of labelled oligonucleotides, one covering the respective area of the xyn2 promoter (Lpxyn2, see Table 1) and the other bearing a mutation in the AGAA-box (from AGAA to CTCC, Lpxyn2 M, see Table 1), were assayed alone (lanes 1, 2), with GST alone (negative control, GST), or with the thrombin-cleaved DBDs (3151prp_DBD, 7236prp_DBD, 2488prp_DBD).

3151prp, $36 \mathrm{kD}$ for 7236prp, and $32 \mathrm{kD}$ for 2488prp). Two clones expressing GST alone were applied as controls and also produced bands of the correct size $(26 \mathrm{kD})$.

The thrombin-cleaved DBD constructs were analyzed with an EMSA using an oligonucleotide covering the whole $x y n 2$ promoter (Lpxyn2, see Table 1) or an oligonucleotide bearing the AGAA-box mutated to CTCC (Lpxyn2 M, see Table 1). FAM-labelled probes seem to demand longer probes: a FAM-labelled (shorter) Pxyn2a only gave a weak shift (data not shown). No shift was observed with the GST control or the 3151prp DBD (Figure 3B). The 7236prp DBD yielded two proteinDNA complexes, but these are rather non-specific, as they also formed with the mutated probe (Figure 3B). In contrast, the 2488prp DBD produced one slow-migrating shift that was not observed with the mutated probe (Figure 3B), indicating the formation of an AGAA-box 
specific DNA-protein complex. This candidate was analyzed in more detail.

\section{The binding of 2488prp to the xyn2 promoter of $H$. jecorina}

Because 2488prp DBD produced an AGAA-box specific shift in the EMSA (Figure 3B), the corresponding gene was subjected to in vitro translation. The accumulation of product from the in vitro translation was confirmed with SDS-PAGE of the FluoroTect ${ }^{\mathrm{Tm}}$ Green-labelled proteins (Figure 4A). A negative control reaction containing no DNA template produced no specific protein, but a positive control reaction containing a plasmid that codes for luciferase gave a protein band of the correct size (61 kD) (Figure 4A). Using the plasmid pMPF2488 as template in an in vitro translation experiment was also found to yield a protein band of the expected size $(54 \mathrm{kD})$ (Figure 4A). Background bands of $42 \mathrm{kD}$ (rabbit reticulocyte lysate protein) and 18 - $25 \mathrm{kD}$ (aminoacyl tRNAs) are associated with the in vitro translation procedure (see manufacturer's guidelines). We then performed an EMSA using the in vitro translated, unlabelled 2488prp and the labelled oligonucleotides (the same as for the EMSA with DBD). No DNA-protein complex formed with the mutated probe (Lpxyn2 M, see Table 1) (Figure

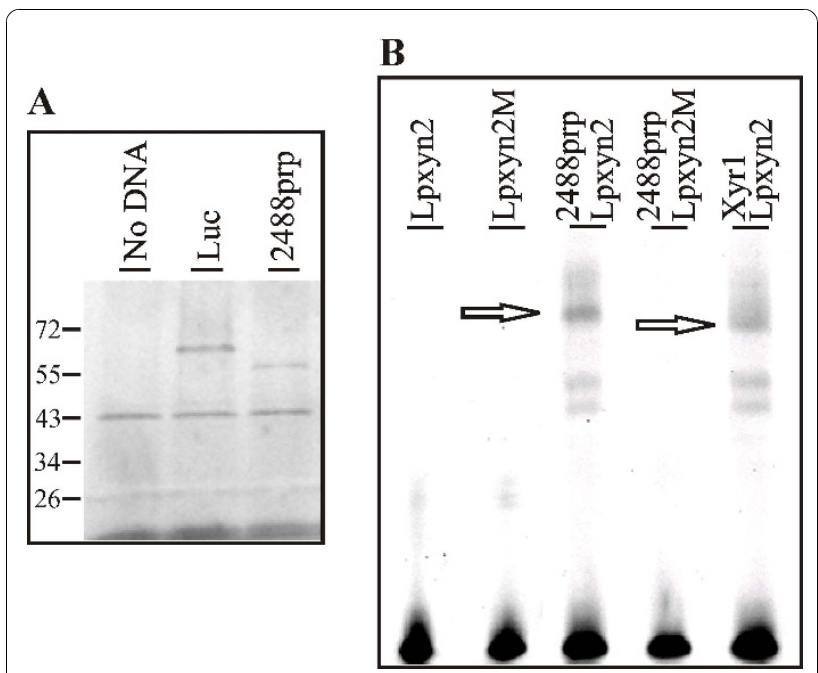

Figure 4 In vitro translation and binding of the $\mathbf{2 4 8 8}$ putative repressor protein. (A) SDS-PAGE of in vitro translated and FluoroTect ${ }^{\mathrm{TM}}$ Green labelled proteins: a negative control reaction containing no DNA template (no DNA), a positive control reaction for the expression of luciferase (Luc), and the expression of 2488prp (pMPF2488 as DNA template). A prestained protein ladder (Fermentas) was applied. (B) EMSA using $60 \mathrm{ng}$ of in vitro translated, unlabelled 2488prp (see Fig. 4A). Then, 15 ng labelled oligonucleotides, one covering the respective area of the xyn2 promoter (Lpxyn2, see Table 1), and another bearing a mutation from AGAA to CTCC (Lpxyn2 M, see Table 1) were applied without protein sample (lanes 1, 2). In vitro translated Xyr1 was used as a positive control.
$4 B)$, but one specific shift was observed with the probe lacking mutations (Lpxyn2) (Figure 4B, indicated by an arrow). This shifted band migrated more slowly than the positive control shifted band using in vitro translated Xyr1 (Figure 4B, also indicated by an arrow). Xyr1, a general hydrolase activating transcription factor in $H$. jecorina [14], was recently reported to bind the xyn2 promoter [18], acting as a cis-acting element near the AGAA-box, which is also present on the applied oligonucleotide. The two shifts with the fastest mobility in the reactions containing oligonucleotide Lpxyn2 and in vitro translated 2488prp or Xyr1 (Figure 4B) are background. These shifts also appear if the in vitro translation mixture is given no DNA-template or the luciferase protein (data not shown). Therefore, we conclude that 2488prp binds the AGAA-box of the $x y n 2$ promoter in vitro.

\section{Characterisation of 2488prp}

The putative 2488prp gene encodes a protein of 505 amino acids with a predicted molecular mass of $55 \mathrm{kD}$. Sequence alignments indicate that the entire 2488prp protein has significant sequence similarity to hypothetical proteins in Nectria haematococca (GeneID: 9678588; 43\%), Gibberella zeae (GeneID: 2791570; 43\%), Neurospora crassa (GeneID: 3874038, 37\%), Podospora anserina (GeneID: 6189947, 37\%) Chaetomium globosum (GeneID: 4395593, 37\%), Magnaporthe grisea (GeneID: $2675104,36 \%)$. The most similarity was found in the Cterminal part of the protein (compare additional file 2), which includes a helix-loop-helix domain (HLH-superfamily; [27]) with an overall length of 67 amino acids (aa 397 to 461). A detailed domain analysis according to [28] revealed a basic DNA binding region (aa 397 to 406) N-terminal to two alpha-helices separated by a loop region (aa 409 to 461 ). The basic N-terminal region is thought to mediate high-affinity DNA-binding [29], whereas the helix-loop-helix region functions as a dimerization interface [30]. A glutamic acid at position 404 strongly indicates that 2488prp belongs to the group of E-box binding HLH proteins (3). The classical E-box is a hexameric palindrome. Interestingly, the AGAA-box overlaps with a hexameric palindrome (TCTAGA on the sense strand). Only the antisense part of this potential E-box has been reported to be protected according to in vivo footprinting experiments $[18,22]$. The sense strand cannot be analyzed because the sequence extension in the linker-mediated PCR is fully determined at the TATA-box (unpublished data, Würleitner E. and Mach R.L.).

\section{Transcription of 2488prp during cultivation in different carbon sources}

In $H$. jecorina, the expression of the hydrolytic enzymeencoding genes, such as $x y n 1, x y n 2, b x l 1, c b h 1$ 
(cellobiohydrolase 1), cbh2 (cellobiohydrolase 2), egl1 (endoglucanase 1$)$, or bgl1 ( $\beta$-glucosidase 1$)$, is regulated by the general activator $\mathrm{Xyr} 1$, regardless of the carbon source or inducing substance [14,31]. Nevertheless, different expression/induction patterns for these genes have been observed $[17,22]$. We examined, whether the transcription of this putative regulatory protein depends on the presence of certain induction signals or various carbon sources.

After pre-cultivation, the mycelium of $H$. jecorina was transferred to medium lacking a carbon source (derepressing conditions) or to media containing $1 \%(\mathrm{w} / \mathrm{v})$ glucose, glycerol (repressing conditions), D-xylose or xylan or $1.5 \mathrm{mM}$ sophorose or xylobiose (inducing conditions). Cultures were incubated for 3, 5, and 24 hours. After RNA-extraction followed by cDNA synthesis, the transcript levels were analyzed via real-time PCR.

We observed that the abundance of the 2488prp transcript increases in the presence of carbon sources that repress hydrolase expression, such as glucose or glycerol, relative to de-repressing conditions (Fig 5A). Similar observations were made when comparing inducing conditions, such as growth on D-xylose or xylan or in media containing xylobiose or sophorose, with transcript forming in media containing glucose (Figure 5B, C). In all cases, it was clear that the transcription of the putative repressor $2488 p r p$ gene was downregulated. These data strongly indicate that 2488prp transcription is upregulated under repressive conditions and that this protein functions as a repressor of hydrolase transcription.

\section{Discussion}

In the last few decades, several techniques have been developed to identify new DNA regulatory elements. Both in vitro methods, such as EMSA with cell-free or nuclear extracts ([32] and citations therein), and in vivo approaches, such as the in vivo genomic footprinting assay $([33,34]$ and citations therein) and promoter deletion analyses, have enabled the identification of numerous cis-acting elements.

The isolation and identification of the corresponding trans-acting factors is still challenging, however. Until recently, affinity chromatography based methods for the isolation of transcription regulators demanded large amounts of samples (e. g. cell-free extract proteins) and/ or laborious pre-purifications steps (e. g. heparin-sepharose purification (e. g. $[35,36])$ ). Furthermore, additional purification steps, such as one- or two-D gel electrophoreses, were necessary to obtain at least semi-purified protein, which could thereafter be subjected to nanospray MS/MS fragmentation sequencing [26]. Although such methods led to the identification of transcription factors, they are labour-intensive and not suitable for highthroughput analyses.

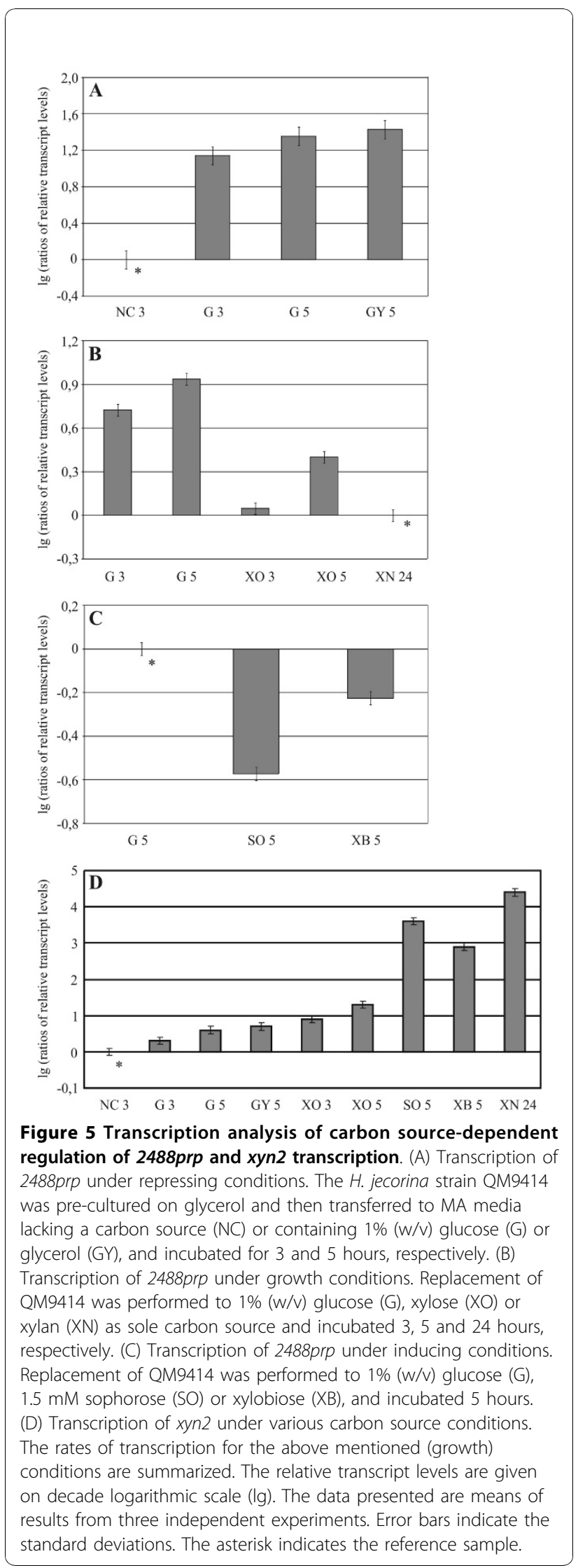


Recently, the yeast one hybrid (Y1H) system was established, offering an in vivo method of screening respective transcription factors in a living cell [37]. This method depends on the completeness of the cDNA library, however. Thus, cDNAs of rare mRNAs, such as those of transcription factors and narrow domain regulators, are often missed. Furthermore, if the DNA binding depends on posttranslational modifications or the presence of additional interacting factors, it is even more likely that relevant interactions can be missed. The $\mathrm{Y} 1 \mathrm{H}$ system is also laborious and time-consuming (e. g. isolation of rare mRNAs, cDNA library constructions) and therefore only partially useful for highthroughput screening approaches. In addition, Y1 $\mathrm{H}$ and $\mathrm{Y} 2 \mathrm{H}$ approaches are known to be prone to false positives (e. g. $[38,39])$.

The method described in this study requires only small amounts of protein extract and the preceding extraction procedure is easy and fast. It combines a single-step micro affinity chromatography purification based on prior in vivo and/or in vitro protein-DNAinteraction data and high-resolution analyses. The Nano-HPLC/tandem MS-coupled detection allows the separation and identification of up to 500 proteins in one sample. Such a large number of identified candidate proteins enable the allocation of protein complexes interacting with the respective DNA target. We required that the putative transcription factor contain a predictable DNA binding domain to be considered for further analyses. Comparing protein extracts derived from different growth or environmental conditions and/or from a time-course experiment could provide information on the dynamics of protein networks interacting with the DNA. Finally, such a technique will permit mutational analysis of cis-acting DNA elements.

In this study, the most promising protein that was identified (2488prp) is an E-box binding HLH protein. The classical E-box is a hexameric palindrome. Interestingly, the AGAA-box overlaps with a hexameric palindrome (TCTAGA on the sense strand). Until this work, only the antisense part of this potential E-box was reported to be protected in footprinting experiments in vivo $[18,22]$, because the sense strand cannot be analyzed due to sequence extension in the linker-mediated PCR is fully determined at the TATA-box (unpublished data, Würleitner E. and Mach R.L).

\section{Conclusions}

The method developed here should be applicable to other high-throughput experiments, given available genome sequence databases. It permits the assignment of a promoter-binding protein (in this study, Xpp1) to its previously identified cis-acting element (the AGAA-box) under various conditions (glucose-repressing conditions) for the expression of a certain gene of interest (xyn2). The described genomic-proteomic approach facilitates the fast one-step isolation and identification of an unknown promoter-binding protein. In addition, this method assists in assigning the promoter-binding protein to a certain gene regulatory function.

\section{Methods}

\section{Strains and growth conditions}

The ascomycete $H$. jecorina ( $T$. reesei) QM9414 (ATCC 26921; a cellulase hyper-producing mutant derived from the wild-type strain QM6a [40]) was used throughout this study and maintained on malt agar. For replacement experiments, mycelia were pre-cultured in 1-L-Erlenmeyer flasks on a rotary shaker $(250 \mathrm{rpm})$ at $30^{\circ} \mathrm{C}$ for $18 \mathrm{~h}$ in $250 \mathrm{~mL}$ of Mandels-Andreotti (MA) medium [41] supplemented with $1 \%(\mathrm{w} / \mathrm{v})$ glycerol as a sole carbon source. $10^{8}$ conidia per litre (final concentration) were used as the inoculum. Pre-grown mycelia were washed and then equal amounts were resuspended in MA media containing 1\% (w/v) glucose, glycerol, xylose or xylan as the sole carbon source or supplemented with $1.5 \mathrm{mM}$ sophorose or xylobiose or without any carbon source.

E. coli JM109 (Promega, Wisconsin, US) was used for the propagation of plasmid vectors, and the strain BL21Gold (Stratagene, La Jolla, CA) was used as the host for the production of GST (Glutathion S-transferase) fusion proteins.

\section{Electrophoretic mobility shift assay (EMSA)}

Radioactive EMSA synthetic oligonucleotides (VBC, Vienna, Austria) (Table 1) were used. After annealing, double stranded oligonucleotides were end-labelled with (*-32P)-dCTP using Klenow Polymerase (Promega) and purified with non-denaturing PAGE (polyacrylamide gel electrophoresis). The binding assays and PAGE experiments were performed essentially as described in [41]. Binding was achieved by incubating $100 \mu \mathrm{g}$ of cell-free extract protein with $5 \mathrm{ng}$ of labelled fragment for 15 min on ice. Cell-free extracts were prepared as described previously [41].

For fluorescent EMSA, the synthetic FAM-labelled oligonucleotides (MWG Biotech, Ebersberg, Germany) were annealed with their complementary oligonucleotides (Table 1) by cooking them in $200 \mathrm{mM}$ Tris/ $\mathrm{HCl}$ ( $\mathrm{pH} \mathrm{7.5)} \mathrm{for} 5 \mathrm{~min}$ and then letting them cool slowly to room temperature. The binding assay and PAGE experiments were performed as described previously [41] Binding was achieved by incubating $100 \mathrm{ng}$ of the GST fusion proteins or $60 \mathrm{ng}$ of in vitro translated, unlabelled 2488prp (putative repressor protein) with $15 \mathrm{ng}$ of labelled fragment for $15 \mathrm{~min}$ on ice. In vitro translation of the complete 2488 prp protein was performed using 
the TNT Quick Coupled Transcription/Translation System (Promega) according to the manufacturer's instructions starting from the plasmid pMPF2488, which inserts the 2488prp structural gene into the vector pTNT (Promega). To verify the completion of the translation process by a SDS-PAGE, both 2488prp and luciferase (a control template DNA provided by Promega) were labelled using FluoroTect ${ }^{\mathrm{Tm}}$ Green (Promega). Gels (EMSA and SDS-PAGE) were analysed using a Typhoon 8600 variable mode imager (Amersham Bioscience, part of GE Healthcare, CT, US).

\section{RNA-extraction, reverse transcription, transcript analyses, quantitative PCR (qPCR)}

Harvested mycelia were homogenized in $1 \mathrm{~mL}$ peqGOLD TriFast DNA/RNA/protein purification system (PEQLAB Biotechnologie, Erlangen, Germany) using a FastPrep FP120 BIO101 ThermoSavant cell disrupter (Qbiogene, Carlsbad, US). RNA was isolated according to the manufacturer's instructions.

The synthesis of cDNA from mRNA was achieved with the RevertAid ${ }^{\text {Tm}} \mathrm{H}$ Minus First Strand cDNA Synthesis Kit (Fermentas, St. Leon-Rot, Germany).

All PCRs for checking transcription were performed in an iCycler iQ, Real-Time Detection System (Bio-Rad, Hercules, US). The reactions were performed in a $25 \mu \mathrm{l}$ volume containing $1 \times$ buffer (Promega), $2.5 \mathrm{mM} \mathrm{MgCl}_{2}$, $0.1 \mu \mathrm{M}$ forward primer, $0.1 \mu \mathrm{M}$ reverse primer, $0.25 \mathrm{U}$ Taq-polymerase (Go-taq, Promega), and a mixture of glucose and xylose-derived cDNAs (10-fold diluted) as template. The primer sequences (transkr2488f/r, transkr3151f/r, transkr3151f/r, transkr7236f/r) are given in Table 1. The PCR run included a blank (sterile, bidistilled water instead of sample). The following PCR protocol was used: $3 \mathrm{~min}$ initial denaturation at $95^{\circ} \mathrm{C}$, followed by 30 cycles of $15 \mathrm{~s}$ at $95^{\circ} \mathrm{C}, 15 \mathrm{~s}$ at $59^{\circ} \mathrm{C}$, and $20 \mathrm{~s}$ at $72^{\circ} \mathrm{C}$.

All qPCRs were performed in a Mastercycler ${ }^{\oplus}$ ep realplex $^{2}$ (Eppendorf, Hamburg, Germany). The software realplex 2.2 was used to compile PCR protocols and define plate set-ups. All reactions were performed in triplicate. To analyze $2488 p r p$ transcription, a SYBR Green assay with an actin reference was performed using $1 \times$ iQ SYBR Green Supermix (Bio-Rad), $0.1 \mu \mathrm{M}$ forward primer, $0.1 \mu \mathrm{M}$ reverse primer, and cDNA as template. Primer pairs are given in Table 1. The following PCR program was used: 3 min initial denaturation at $95^{\circ} \mathrm{C}$, followed by 45 cycles of $15 \mathrm{~s}$ at $95^{\circ} \mathrm{C}, 15 \mathrm{~s}$ at $59^{\circ} \mathrm{C}$ and $15 \mathrm{~s}$ at $72^{\circ} \mathrm{C}$. The data are expressed relative to the transcription of the actin gene. The data in the figures are means of three independent experiments. Error bars indicate standard deviations. These amounts always refer to one reference sample within an experiment, which is marked in the figure with an asterisk.

\section{Isolation of DNA-binding proteins with streptavidin affinity chromatography}

Biotinylated oligonucleotides (VBC, Vienna, Austria) (Bxyn2p250f/r, see Table 1) were annealed $\left(95^{\circ} \mathrm{C}, 5 \mathrm{~min}\right.$ followed by a slow cool to $35^{\circ} \mathrm{C}$ and then ice) and incubated with $200 \mu \mathrm{L} \mu \mathrm{MACS}$ Streptavidin Micro Beads (Miltenyi Biotec GmbH, Bergisch Gladbach, Germany) on ice for $15 \mathrm{~min}$. Afterwards, $800 \mu \mathrm{L}$ of binding mix (200 mM KCl, $200 \mathrm{ng} / \mu \mathrm{L}$ poly $(\mathrm{dIdC}), 200 \mathrm{ng} / \mu \mathrm{L}$ CKT067/CKT068, $8 \mathrm{mM}$ Spermidine) and then $880 \mu \mathrm{L}$ of cell-free extract protein were added and incubated on ice for $15 \mathrm{~min}$ first and then for $10 \mathrm{~min}$. CKT067/ CKT068 is a double-stranded DNA fragment used to titrate proteins that nonspecifically interact with DNA. This fragment and poly $(\mathrm{dIdC})$ is used in a ten-fold excess of the specific probe [41]. Cell-free extracts were prepared as described previously [41]. Magnetic separation was performed according to the manufacturer's instructions after equilibration and subsequent saturation with CKT067/068 was achieved. Elution was performed with $150 \mu \mathrm{L}$ of cold (room temperature) SDS loading buffer followed by $150 \mu \mathrm{L}$ of hot $\left(60\right.$ to $\left.70^{\circ} \mathrm{C}\right)$ SDS loading buffer. The eluate was placed on ice immediately.

\section{Nano-HPLC-separation and identification of target proteins}

Prior to MS analysis, reversed phase (RP) nano-HPLC separation of the eluted peptide mixtures from an in gel digest was performed. An UltiMate ${ }^{\text {TM }}$ HPLC System equipped with a FAMOS autosampler, Switchos and UV detector (all Dionex, Sunnyvale, CA, US) was used. After concentration and desalting on a trapping column (Acclaim PepMap100 C18, $5 \mu \mathrm{m}, 100 \AA$, $300 \mu \mathrm{m}$ i.d. $\times 5$ mm, Dionex), peptides were separated on a PepMap 100 (C18) nanocolumn (Acclaim PepMap100 C18, $3 \mu \mathrm{m}$, $100 \AA 75 \mu \mathrm{m}$ i.d. $\times 15 \mathrm{~cm}$, Dionex) at a flow-rate of 275 $\mathrm{nL} / \mathrm{min}$. Peptides were eluted with a linear gradient from $0 \%$ to $50 \% \mathrm{~B}$ in $30 \mathrm{~min}$ formed by mixing the two solvents A (5\% ACN, 0.1\% FA) and B (80\% ACN, $0.08 \%$ FA), followed by a high organic wash (4 min at $90 \% \mathrm{~B}$ ). The quality of separation was monitored by UV absorption at $214 \mathrm{~nm}$.

The outlet of the nano-HPLC system was directly coupled to a Thermo ${ }^{\mathrm{Tm}} \mathrm{LTQ}$ linear ion trap mass spectrometer (Thermo Electron Corp., Waltham, MA, US). Mass spectra were acquired in positive ionization mode. The applied method consisted of seven scans; the first was used to determine the precursor ions that were investigated in the following tandem mass scans by CAD (collisionally activated dissociation) fragmentation.

\section{Database searching}

Tandem mass spectra were extracted by extract-msn (Thermo). Charge state deconvolution and deisotoping 
were not performed. All MS/MS samples were analyzed using Mascot (Matrix Science, London, UK; version 2.2.04). Mascot was set up to search the treeseiV2_FrozenGeneCatalog20081022.proteins.fasta.gz database (unknown version, 9143 entries), using predictions for a trypsin digest. Mascot was searched with a fragment ion mass tolerance of $0.60 \mathrm{Da}$ and a parent ion tolerance of 1.5 Da. The iodoacetamide derivative of cysteine was specified in Mascot as a fixed modification. S-carbamoylmethylcysteine cyclization of the $\mathrm{N}$-terminus and methionine oxidation were specified in Mascot as variable modifications.

\section{Criteria for protein identification}

Scaffold (version Scaffold-01_07_00, Proteome Software Inc., Portland, OR) was used to validate MS/MS based peptide and protein identifications. Peptide identifications were accepted if they could be established at greater than $95.0 \%$ probability as specified by the Peptide Prophet algorithm [42]. Protein identifications were accepted if they could be established at greater than 99.0\% probability and contained at least three identified peptides. Protein probabilities were assigned by the Protein Prophet algorithm [43]. Proteins that contained similar peptides and could not be differentiated based on MS/MS analysis alone were grouped to satisfy the principles of parsimony.

\section{Vector construction}

Vectors for expressing the DNA-binding domains (DBD) as GST fusion proteins were constructed as follows. PCR was used to amplify the DBD coding regions using $25 \mu$ reaction-mixtures containing $1 \times$ buffer with $\mathrm{MgCl}_{2}$ (Fermentas), $0.1 \mu \mathrm{M}$ forward primer, $0.1 \mu \mathrm{M}$ reverse primer, $0.5 \mathrm{U}$ High Fidelity DNA Polymerase (Fermentas), and genomic DNA as the template. Primer sequences (Exp2488F/R, Exp3151F/R, and Exp7236F/R) are given in Table 1 . The following PCR protocol was followed: $3 \mathrm{~min}$ initial denaturation at $95^{\circ} \mathrm{C}$, followed by 30 cycles of $15 \mathrm{~s}$ at $95^{\circ} \mathrm{C}, 15 \mathrm{~s}$ at $59^{\circ} \mathrm{C}$, and $15 \mathrm{~s}$ at $72^{\circ} \mathrm{C}$. Derived amplicons (165 bp, $219 \mathrm{bp}$, and $258 \mathrm{bp}$ ) were inserted into plasmid pGEX-4T-2 (Amersham) via the restriction enzymes BamHI and XhoI to obtain p2488DBD, p3151DBD, and p7236DBD, respectively.

The plasmid for expressing the 2488prp full length protein as an in vitro translation product was constructed as follows. PCR was used to amplify full length cDNA as described above, but in this case, the template was a mixture of cDNAs obtained under repressing conditions (glucose). The primer sequences (fltn2488f/r) are given in Table 1. The PCR protocol was as follows: 3 min initial denaturation at $95^{\circ} \mathrm{C}$, followed by 30 cycles of $15 \mathrm{~s}$ at $95^{\circ} \mathrm{C}, 15 \mathrm{~s}$ at $59^{\circ} \mathrm{C}$, and $2 \mathrm{~min}$ at $72^{\circ} \mathrm{C}$. The derived amplicon (1,479 bp) was inserted into plasmid pTNT (Promega) via the restriction enzyme KpnI and NotI to obtain pMPF2488.

\section{Purification of the DBDs of 2488prp, 3151prp, and 7236 prp as GST fusion proteins}

All heterologously expressed proteins used in this study were produced as GST fusions with the pGEX system (Amersham), following the manufacturer's guidelines and using the plasmids p2488DBD, p3151DBD, and p7236DBD as templates. Expression products purified via glutathione-sepharose-columns (Amersham) were verified by SDS-PAGE (using the Mini-PROTEAN System, Bio-Rad) followed by SYPRO Ruby (Bio-Rad) staining. Thrombin cleavage was performed for $1 \mathrm{~h}$ at $37^{\circ} \mathrm{C}$ with $1 \mathrm{U}$ of thrombin (Amersham), $150 \mathrm{mM} \mathrm{NaCl}, 2.5$ $\mathrm{mM} \mathrm{CaCl}_{2}$, and $25 \mathrm{mM}$ Tris/ $\mathrm{HCl}(\mathrm{pH} 8)$ plus $20 \%$ Glycerin to remove the GST moiety.

\section{Additional material}

Additional file 1: List of $\boldsymbol{H}$. jecorina proteins identified by tandem MS analysis. The corresponding proteomic data are available at the publically available database https://proteomecommons.org/.

Additional file 2: Multiple sequence alignment of the $\mathrm{H}$. jecorina 2488prp with hypothetical proteins of other fungi, namely Nectria haematococca (GeneID: 9678588), Gibberella zeae (GenelD:

2791570), Neurospora crassa (GenelD: 3874038), Podospora anserina (GenelD: 6189947) Chaetomium globosum (GenelD: 4395593), Magnaporthe grisea (GeneID: 2675104).

\section{Acknowledgements}

We wish to thank Alice Rassinger for experimental support. This work was supported by a grant from the Austrian Science Fund ( $P$ 20192-B03) given to RLM and by the Austrian Proteomics Platform (APP) within the Austrian Genome Research Program (GEN-AU) given to KM, which is gratefully acknowledged. MJPF was supported by a post-doctoral fellowship from CAPES (Coordenação de Aperfeiçoamento de Pessoal de Nível Superior) - Brazil, which is also gratefully acknowledged.

\section{Author details}

${ }^{1}$ Gene Technology, Department of Gene Technology and Applied Biochemistry, Institute of Chemical Engineering, TU Wien, Getreidemarkt 9/166/5/2, A-1060 Wien, Austria. 2Department of Genetics and Morphology, Institute of Biological Sciences, University of Brasilia, Campus Universitário Darcy Ribeiro, ICC SuL, AT-098, 70.910-900, Brasília-DF, Brazil. ${ }^{3}$ Research Institute of Molecular Pathology, Vienna Biocenter, Dr. Bohrgasse 7, A-1030 Vienna, Austria.

\section{Authors' contributions}

ARMA carried out the EMSAs, streptavidin affinity chromatography assays, transcript analyses, and drafted the manuscript. KGH carried out the NanoHPLC/tandem MS-coupled detection and participated in the database search. MJPF carried out the vector construction and expression of GST fusion proteins. KM participated in the database search and data interpretation. RLM participated in the conception of the study and revised the manuscript. All authors read and approved the final manuscript

Received: 23 June 2010 Accepted: 18 November 2010 Published: 18 November 2010 


\section{References}

1. Kuhls K, Lieckfeldt E, Samuels GJ, Kovacs W, Meyer W, Petrini O, Gams W, Borner T, Kubicek CP: Molecular evidence that the asexual industrial fungus Trichoderma reesei is a clonal derivative of the ascomycete Hypocrea jecorina. Proc Natl Acad Sci USA 1996, 93(15):7755-7760.

2. Nidetzky B, Steiner W, Hayn M, Claeyssens M: Cellulose hydrolysis by the cellulases from Trichoderma reesei: a new model for synergistic interaction. Biochem J 1994, 298 Pt 3:705-710.

3. Woodward J, Lima M, Lee NE: The role of cellulase concentration in determining the degree of synergism in the hydrolysis of microcrystalline cellulose. Biochem J 1988, 255(3):895-899.

4. Ward M: Improving secreted Enzyme Production by Trichoderma reesei. 9th International Workshop on Trichoderma and Gliocladium: 2006; Vienna 2006.

5. Miettinen-Oinonen A, Suominen P: Enhanced production of Trichoderma reesei endoglucanases and use of the new cellulase preparations in producing the stonewashed effect on denim fabric. Appl Environ Microbiol 2002, 68(8):3956-3964.

6. Harbord R, Simpson C, Wegstein J: Winery scale evaluation of macerating enzymes in grape processing. Wine industry J 1990, 134-137.

7. Servili M, Begliomini AL, Montedoro G: Utilization of a yeast pectinase in olive oil extraction and red wine making processes. J Sci Food Agric 1992 58:253-260.

8. Walsh GA, Power RF, Headon DR: Enzymes in the animal-feed industry. Trends Biotechnol 1993, 11(10):424-430.

9. Oksanen T, Pere J, Paavilainen L, Buchert J, Viikari L: Treatment of recycled kraft pulps with Trichoderma reesei hemicellulases and cellulases. J Biotechnol 2000, 78(1):39-48.

10. Teeri TT, Penttilä M, Keränen S, Nevalainen H, Knowles JKC: Biotechnology of Filamentous Fungi: Technology and Products Boston, MA: ButterworthHeinemann; 1992.

11. Hahn-Hägerdal B, Galbe M, Gorwa-Grauslund MF, Liden G, Zacchi G: Bioethanol-the fuel of tomorrow from the residues of today. Trends in biotechnology 2006, 24(12):549-556.

12. Himmel ME, Ding SY, Johnson DK, Adney WS, Nimlos MR, Brady JW Foust TD: Biomass recalcitrance: engineering plants and enzymes for biofuels production. Science (New York, NY 2007, 315(5813):804-807.

13. Ragauskas AJ, Williams CK, Davison BH, Britovsek G, Cairney J, Eckert CA, Frederick WJ, Hallett JP, Leak DJ, Liotta $C L$, et al: The path forward for biofuels and biomaterials. Science (New York, NY 2006, 311(5760):484-489.

14. Stricker AR, Grosstessner-Hain K, Würleitner E, Mach RL: Xyr1 (xylanase regulator 1) regulates both the hydrolytic enzyme system and D-xylose metabolism in Hypocrea jecorina. Eukaryot Cell 2006, 5(12):2128-2137.

15. Aro N, Saloheimo A, Ilmen M, Penttilä M: ACEIl, a novel transcriptional activator involved in regulation of cellulase and xylanase genes of Trichoderma reesei. J Biol Chem 2001, 276(26):24309-24314.

16. Saloheimo A, Aro N, IImen M, Penttilä M: Isolation of the ace1 gene encoding a Cys(2)-His(2) transcription factor involved in regulation of activity of the cellulase promoter cbh1 of Trichoderma reesei. J Biol Chem 2000, 275(8):5817-5825.

17. Rauscher R, Würleitner E, Wacenovsky C, Aro N, Stricker AR, Zeilinger S, Kubicek CP, Penttilä M, Mach RL: Transcriptional regulation of xyn 1, encoding xylanase I, in Hypocrea jecorina. Eukaryot Cell 2006, 5(3):447-456.

18. Stricker AR, Trefflinger P, Aro N, Penttilä M, Mach RL: Role of Ace (Activator of Cellulases 2) within the xyn2 transcriptosome of Hypocrea jecorina. Fungal Genet Biol 2008, 45(4):436-445.

19. Strauss J, Mach RL, Zeilinger S, Hartler G, Stoffler G, Wolschek M, Kubicek CP: Cre1, the carbon catabolite repressor protein from Trichoderma reesei. FEBS Lett 1995, 376(1-2):103-107.

20. Ilmen M, Thrane C, Penttilä M: The glucose repressor gene cre1 of Trichoderma: isolation and expression of a full-length and a truncated mutant form. Mol Gen Genet 1996, 251(4):451-460

21. Mach RL, Strauss J, Zeilinger S, Schindler M, Kubicek CP: Carbon catabolite repression of xylanase I (xyn1) gene expression in Trichoderma reesei. Mol Microbiol 1996, 21(6):1273-1281.

22. Würleitner E, Pera L, Wacenovsky C, Cziferszky A, Zeilinger S, Kubicek CP, Mach RL: Transcriptional regulation of xyn2 in Hypocrea jecorina. Eukaryot Cell 2003, 2(1):150-158

23. Geyer $H$, Geyer $R$, Pingoud V: A novel strategy for the identification of protein-DNA contacts by photocrosslinking and mass spectrometry. Nucleic Acids Res 2004, 32(16):e132.
24. Liu J, Stormo GD: Combining SELEX with quantitative assays to rapidly obtain accurate models of protein-DNA interactions. Nucleic Acids Res 2005, 33(17):e141.

25. Djordjevic M: SELEX experiments: new prospects, applications and data analysis in inferring regulatory pathways. Biomol Eng 2007, 24(2):179-189.

26. Samuel S, Twizere JC, Bernstein LR: YB-1 represses AP1-dependent gene transactivation and interacts with an AP-1 DNA sequence. Biochem J 2005, 388(Pt 3):921-928.

27. Anthony-Cahill SJ, Benfield PA, Fairman R, Wasserman ZR, Brenner SL, Stafford WF, Altenbach C, Hubbell WL, DeGrado WF: Molecular characterization of helix-loop-helix peptides. Science 1992, 255(5047):979-983.

28. Marchler-Bauer A, Anderson JB, Derbyshire MK, DeWeese-Scott C Gonzales NR, Gwadz M, Hao L, He S, Hurwitz DI, Jackson JD, et al: CDD: a conserved domain database for interactive domain family analysis. Nucleic Acids Res 2007, 35 Database: D237-240.

29. Ledent $V$, Vervoort M: The basic helix-loop-helix protein family: comparative genomics and phylogenetic analysis. Genome Res 2001, 11(5):754-770

30. Chavali GB, Vijayalakshmi C, Salunke DM: Analysis of sequence signature defining functional specificity and structural stability in helix-loop-helix proteins. Proteins 2001, 42(4):471-480.

31. Stricker $A R$, Steiger MG, Mach RL: Xyr1 receives the lactose induction signal and regulates lactose metabolism in Hypocrea jecorina. FEBS Lett 2007, 581(21):3915-3920

32. Lane $D$, Prentki $P$, Chandler M: Use of gel retardation to analyze protein nucleic acid interactions. Microbiol Rev 1992, 56(4):509-528.

33. Saluz HP, Wiebauer K, Wallace A: Studying DNA modifications and DNAprotein interactions in vivo. A window onto the native genome. Trends Genet 1991, 7(7):207-211.

34. Hornstra IK, Yang TP: In vivo footprinting and genomic sequencing by ligation-mediated PCR. Anal Biochem 1993, 213(2):179-193.

35. Borensztajn J, Kotlar TJ, Meredith SC: Fractionation of chylomicrons by heparin-sepharose chromatography. Characterization of two heparinbinding proteins. J Biol Chem 1985, 260(24):13047-13052

36. Srivastava PN, Farooqui AA: Heparin-sepharose affinity chromatography for purification of bull seminal-plasma hyaluronidase. Biochem J 1979, 183(3):531-537.

37. Wang MM, Reed RR: Molecular cloning of the olfactory neuronal transcription factor Olf-1 by genetic selection in yeast. Nature 1993 , 364(6433):121-126.

38. Deplancke B, Dupuy D, Vidal M, Walhout AJ: A gateway-compatible yeast one-hybrid system. Genome Res 2004, 14(10B):2093-2101.

39. Lopato S, Bazanova N, Morran S, Milligan AS, Shirley N, Langridge P: Isolation of plant transcription factors using a modified yeast one-hybrid system. Plant Methods 2006, 2:3.

40. Mandels M: Applications of cellulases. Biochem Soc Trans 1985 13(2):414-416.

41. Stangl H, Gruber F, Kubicek CP: Characterization of the Trichoderma reesei cbh2 promoter. Curr Genet 1993, 23(2):115-122.

42. Keller A, Nesvizhskii Al, Kolker E, Aebersold R: Empirical statistical model to estimate the accuracy of peptide identifications made by MS/MS and database search. Anal Chem 2002, 74(20):5383-5392

43. Nesvizhskii Al, Keller A, Kolker E, Aebersold R: A statistical model for identifying proteins by tandem mass spectrometry. Anal Chem 2003, 75(17):4646-4658

\section{doi:10.1186/1471-2164-11-644}

Cite this article as: Mach-Aigner et al.: From an electrophoretic mobility shift assay to isolated transcription factors: a fast genomic-proteomic approach. BMC Genomics 2010 11:644. 